\title{
Espacios urbanos, entormos multiusuarios y su interconectividad en la web
}

\author{
Urban spaces, multiuser surroundings \\ and their interconnectivity in the web
}

Jane J. EspinaB. ${ }^{*}+$ Javier Oliva**

$<$ Resumen>

El presente artículo plantea el uso de mundos virtuales multiusuarios, como herramienta en procesos de planificación e intervención urbana en el área profesional y en la docencia, basado en una investigación desarrollada en el contexto de la Plaza Baralt de Maracaibo.

$<$ Abstract>

The present article outlines the use of virtual multi-user worlds, as a tool in urban planning and interventions in the professional and teaching areas, based on a research developed in the context of the Plaza Baralt of Maracaibo.

<PALABRAS CLAVE>

MUNDOS VIRTUALES MULTIUSUARIOS / PLANIFICACIÓN

URBANA / DOCENCIA

KKEY WORDS

KKEY WORDS > TEACHING

\section{Introducción}

En la actualidad, los avances tecnológicos proveen de nuevos recursos o medios digitales que pueden lograr beneficios en la planificación y diseño de espacios urbanos. La incorporación de estas técnicas en el ámbito profesional y en el educativo puede aportar beneficios significativos, ya que su uso en la recreación y simulación de espacios urbanos, permite la interactividad y el trabajo colaborativo y multidisciplinar, producto de la combinación de procesos estéticos, técnicos, espaciales, funcionales y económicos, entre otros.

En la investigación, se plantea la incorporación de herramientas para la planificación y diseño de espacios urbanos, que pueden ser utilizadas a futuro en la enseñanza del urbanismo en nuestro contexto regional, permitiéndoles a los docentes desarrollar, optimizar y evaluar las propuestas de los estudiantes en la solución de los problemas de diseño planteados.

La herramienta digital se encuentra en fase de desarrollo, está constituida por modelos tridimensionales virtuales del espacio urbano, con elementos de ambientación coincidentes con las actividades propias del sitio, incorporando el uso de avatares, sonidos, videos y animaciones. Se hace uso de un entorno multiusuario como base para el funcionamiento y la edición interactiva.

\section{Antecedentes}

En la temática objeto de estudio, no se conocen antecedentes previos en el país. Se han realizado trabajos sobre la

\footnotetext{
* Profesora, Arquitecto Dra., Facultad de Arquitectura y Diseño, Universidad del Zulia, Maracaibo, Venezuela

** Profesor, Arquitecto, Facultad de Arquitectura y Diseño, Universidad del Zulia, Maracaibo, Venezuela.
} 
reconstrucción de la Plaza Baralt ${ }^{1}$, generación de mundos multiusuarios ${ }^{2}$, creación de entornos virtuales ${ }^{3}$; utilización de métodos de planificación, participación ciudadana monitoreo en línea en ambientes de simulación y recorrido interactivo por escenarios virtuales ${ }^{4}$; y técnicas de trabajo colaborativo para visualizar e interactuar en la planificación urbana, en la que arquitectos o ingenieros pueden plantear propuestas, discutirlas y modificar sus proyectos ${ }^{5}$

\section{Objetivo}

En esta investigación se plantea, como objetivo general, utilizar los entornos multiusuarios como herramienta para el diseño, la planificación y trabajo en redes en espacios urbanos de carácter patrimonial.

\section{Espacios urbanos versus Entormos virtuales}

Los espacios urbanos son entes vivos y complejos, centros de la vida urbana, producto de la creación de sus habitantes y actores en interacción, usos, vivencias y el significado que los ciudadanos le otorgan. Estos espacios evolucionan o se transforman con el transcurrir de los años. En algunos casos, su morfología no es producto de una planificación, sino el resultado de un conjunto de actividades y usos del suelo.

La Plaza Baralt, es un espacio urbano colectivo y dinámico con características especiales, de origen colonial y carácter patrimonial, localizado en el centro histórico de la ciudad de Maracaibo. Ha experimentado continuas transformaciones urbanas en diferentes períodos históricos, con la construcción, remodelación y demolición de edificios. En la actualidad, está delimitado en su contexto inmediato entre el Templo San Francisco y la Avenida Libertador.

Es importante destacar, que en este tipo de espacios urbanos, se hace difícil plantear nuevos diseños en los aspectos formales y espaciales, debido a las restricciones vigentes, y a las características especiales que poseen por formar parte del patrimonio de la ciudad. Por ende, las propuestas se realizarían sólo en el mobiliario urbano y el planteamiento de nuevos usos del suelo.

El uso de nuevas herramientas digitales en la lectura del fenómeno urbano y sus transformaciones por parte de especialistas es cada vez es mayor, en especial en la producción de modelos urbanos en 3D, basados en tecnologías que permiten recorridos virtuales en tiempo real.

Es allí, donde los entornos virtuales interactivos se convierten en una herramienta poderosa, para viabilizar el desarrollo o planificación de los cambios pertinentes en la realidad urbana, mostrando los resultados de las simulaciones en tiempo real, facilitando así el análisis y la toma de decisiones. Por otro lado, proveen un eficaz medio de visualización, ya que los usuarios sienten la ilusión de estar totalmente rodeados de información, adquiriendo conciencia de otros usuarios que podrían estar conectados, con una significativa sensación de inmersión, presencia y un elevado nivel de interactividad.

La generación de estos mundos virtuales es un esfuerzo multidisciplinar; proporciona una herramienta eficaz en la elaboración de propuestas y el estudio de los efectos de las intervenciones de tipo urbano, siendo casi ilimitado el conjunto de mundos que pueden ser generados y estudiados, los períodos de tiempo que el usuario puede navegar, y las situaciones que en las cuales puede participar, todo esto con un elevado nivel de interactividad.

\section{Ambientes multiusuario}

Un mundo virtual multiusuario es básicamente un conjunto de objetos y situaciones con diferentes niveles de interacción y comportamientos que admite la visita simultánea e interactiva de varios participantes, a través de la Internet o en una Intranet, pudiendo cada uno de ellos estar representado por un avatar humanoide o no ${ }^{6}$

La inclusión del cuerpo humano mediante una representación digital en los modelos recorridos es importante desde el punto de vista de la percepción de la escala de los espacios, en lo referente a la ambientación y caracterización de los mismos con relación a los usos, actividades e interacción de los usuarios ${ }^{7}$.

Al recorrer modelos arquitectónicos utilizando representaciones con forma humana, los usuarios pueden proyectarse mentalmente dentro de sus representaciones digitales, y al mismo tiempo ver a sus avatares desde el exterior, lo cual constituye una forma de alienación, necesaria cuando se trata de un proceso autocrítico, como pudiera serlo el recorrer modelos diseñados por uno mismo con fines de corrección y mejoramiento ${ }^{8}$.

La selección del entorno multiusuario a utilizar, estuvo basada en el trabajo de investigación titulado «Implementación de un Sistema RV Multiusuario en los Laboratorios de la FAD-LUZ. Caso: DEPG», del Prof. Javier Oliva, en el cual se realizó un proceso de estudio de los entornos multiusuarios más populares, para seleccionar el que mejor se adaptara a las necesidades específicas y a la configuración de hardware, software y red existente en la División de Estudios para Graduados de la Facultad de Arquitectura y Diseño.

Luego de este estudio, se decidió utilizar el VRSpace, sistema Cliente - Servidor de código abierto y licencia GPL, para incorporar múltiples usuarios en la visualización de los modelos 3D. Desarrollado en Java, permite el Chat y la modificación de los objetos en el mundo virtual; funciona con los plugin y navegadores más utilizados para VRML ${ }^{9}$.

De este modo la interfaz del programa se utilizaría en función de los objetivos planteados

Espina, J. «Lo intangible y real del espacio urbano Plaza Baralt». VIII Congreso Iberoamaericano de Gráfica Digital, 2004

Espina, J. «Visión o retrospección del pasado: Visualización científica en la recuperación de espacios urbanos patrimoniales». IX Congreso Iberoamericano de Gráfica Digital, 2005. Bustos, G.; Burgos, I.; Oliva, J. «Mundos multiusuario: Visualización 3D interactiva en ambientes sintéticos en los talleres de diseño». IX Congreso Iberoamericano de Gráfica Digital, 2005

3 Perales, F. et al. El Proyecto INEVAI 3D: Agentes autónomos 3D, escenarios virtuales e interfaces inteligentes para aplicaciones de domótica y de realidad virtual, 2002. http:// dmi.uib.es/research/GV/INEVAI3D/publicacion/ucami_inevai_final.pdf [consultada 01-05-2006].

4 Voigt, A.; Peter H.; Linzer H. «City experimental lab». IV Seminário Iberoamericano de Gráfica Digital, 2000.

5 Manoharan, T.; Taylor H.; Gardiner P. A Collaborative Analysis Tool for Visualisation and Interaction with Spatial Data, 2002. http://portal.acm.org/citation.cfm?id=504515\&dl=AC M\&coll=portal\&CFID=11111111\&CFTOKEN=2222222 [consultada: 01-10-2006].

6 Vélez, G. «Arquitectura virtual: Fronteras». IV Congreso Iberoamericano de Gráfica Digital, 2000.

Oliva, J. «Implementación de un Sistema RV Multiusuario para los Laboratorios de la FADLUZ. Caso: DEPG». Tesis de Maestría. Universidad del Zulia, Facultad de Arquitectura y Diseño, 2006.

Oliva, J., op. cit.

9 VRSpace.org. Documentation. http://www.vrspace.org/index1.html [consultada: 16-03-2005]. 
para las propuestas de intervención urbana, permitiendo la simulación de las condiciones reales de los espacios a intervenir.

\section{Interconectividad en la web}

En los procesos de diseño colaborativo, el papel de las Intranets y la Internet se hace determinante, puesto que permiten la participación de profesionales, asesores y usuarios ubicados en diferentes localidades, durante el desarrollo de propuestas en todas las escalas de diseño, incluyendo el urbanismo.

El acceso al sistema multiusuario, se establece mediante un hipervínculo en la página web del proyecto, permitiendo el acceso a la información teórica, gráfica y multimedia, especialmente los modelos 3D interactivos en VRML, en modo multiusuario o monousuario.

Esto permite que los datos se encuentren disponibles para los profesionales autorizados mediante la asignación de un login y una contraseña, quienes tendrán la posibilidad de modificar los mundos virtuales para el estudio de sus propuestas. Por otro lado, es posible mantener en el sistema logins y contraseñas predefinidas para usuarios de tipo público, que podrán visualizar el contenido de los mundos virtuales, pero no modificarlos.

Herramienta para la Práctica Profesional o la Docencia

La complejidad del hecho urbano, el surgimiento de nuevas actividades y los cambios en la lectura de la ciudad, requieren de nuevos métodos y un trabajo multidisciplinar efectivo para lograr los mejores resultados en la planificación y diseño de un proyecto urbano. Asimismo, se requieren nuevos medios en la transmisión del conocimiento de los docentes a los estudiantes, para lograr los resultados esperados al final de las asignaturas dictadas, de manera que el proceso de enseñanzaaprendizaje sea fluido, dinámico, participativo y que los ejercicios realizados en la creación de espacios públicos o sectores urbanos, originen resultados eficientes en el menor tiempo posible.

Evidentemente, con los métodos tradicionales de enseñanza, no es tan fácil lograrlo. ¿Cómo generar nuevas pautas para un mejor diseño urbano, en el cual lo pensado y creado, pueda ser modificado cuantas veces sea necesario y que permitan a la vez la participación de varios estudiantes y profesores
Vistas del mundo virtual de la Plaza Baralt

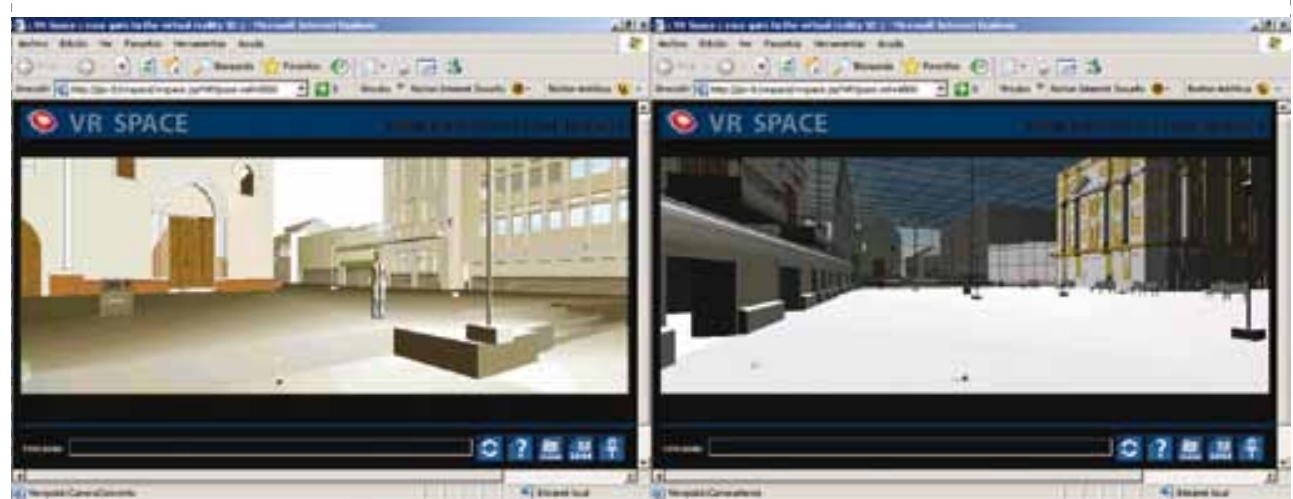

Vistas del mundo virtual multiusuario: Interfaz de edición.

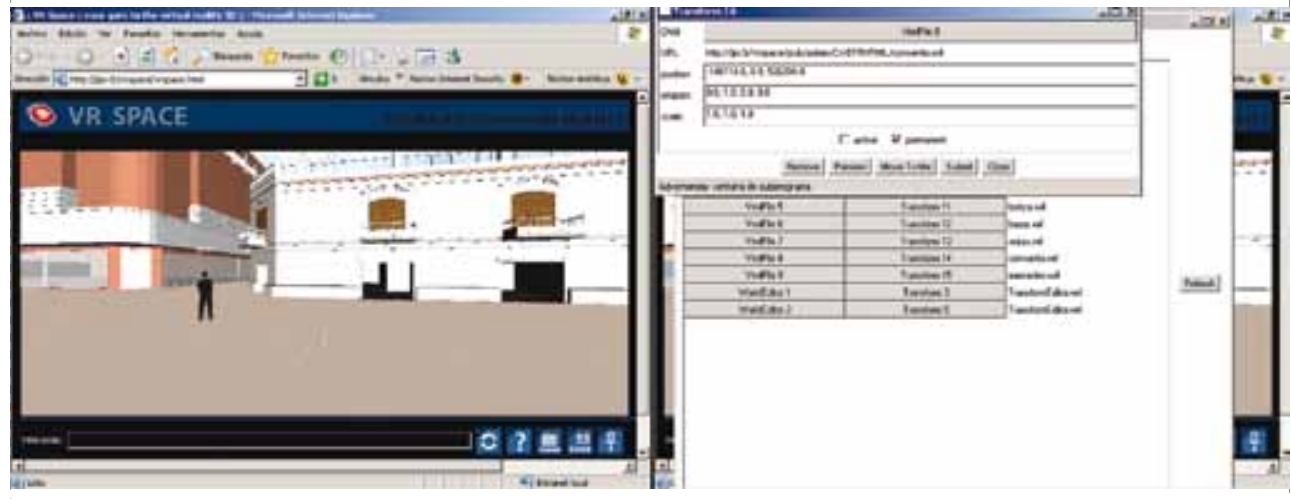

Vistas de la página web del proyecto
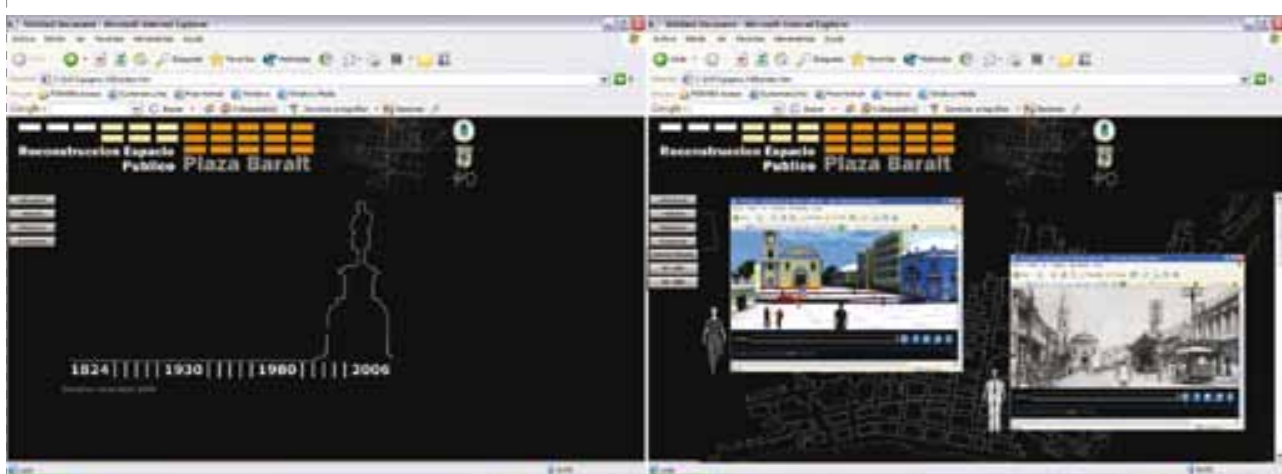
en la dinámica de planteamiento de ideas y soluciones? Es recomendable entonces, el diseñar y proponer nuevas metodologías que incluyan el uso de herramientas digitales para la enseñanza del urbanismo, y de esa forma lograr los objetivos planteados.

Es de hacer notar, que se trata de generar nuevas herramientas para mejorar la percepción y diseño del espacio urbano, en combinación con las nuevas tecnologías de visualización. La utilización de los entornos virtuales, permitirá explorar y desarrollar varias alternativas o propuestas de diseño, con las cuales se podrá interactuar para realizar labores de edición y seleccionar así la más adecuada. Estas técnicas digitales pueden ser empleadas no sólo en las fases finales de diseño, para mostrar el proyecto, sino también en instancias previas a la concreción de mismo, como por ejemplo, en la generación de las ideas, relaciones, morfología, características espaciales, entre otras.

Cabe señalar, que en la investigación se utilizarán los entornos virtuales y los ambientes multiusuarios resultantes del proyecto intitulado «Reconstrucción del Espacio Público Urbano: Plaza Baralt», que actualmente está en ejecución, los cuales serán incorporados en el dictado de las cátedras de urbanismo en las primeras fases del proceso de diseño. Ésta constituye la primera experiencia en la ciudad, en la cual se incorporan medios virtuales colaborativos en el diseño de espacios urbanos.

Esto no significa, claro está, una sustitución de los procesos y prácticas tradicionales, sino la incorporación de herramientas digitales en las distintas etapas del proceso de diseño, para su mejoramiento y enriquecimiento. Además, el proceso de visualización a través de medios digitales, estimulará la adquisición y desarrollo de la memoria-creativa de los estudiantes participantes en proyectos de arquitectura y urbanismo.
Un aporte adicional de esta herramienta, es la realización de sesiones de trabajo colaborativo, trabajando en redes, a través de la Intranet o Internet. También, es importante reflexionar sobre la aplicación de ésta en el proceso creativo de diseño y no sólo como un instrumento de representación.

\section{Espacios urbanos con tecnologías de avanzada}

La implantación de estas tecnologías en el proceso de planificación y diseño urbano, incrementará el nivel de participación de usuarios con experiencia profesional o con escasos conocimientos en el área, como pueden ser los estudiantes de arquitectura en los inicios de su carrera, ya que pueden incorporarse de manera intuitiva, mediante eventos en la web, o en la Intranet. Esto facilitaría las consultas, la planificación en forma conjunta y el planteamiento de soluciones a los diferentes problemas planteados.

La herramienta planteada proporcionaría un instrumento fundamental para una intervención multidisciplinar que implique la visión, síntesis y discusión de escenarios propuestos, verificando en tiempo real cualquier tipo de errores en su diseño. La creación de entornos virtuales genera la capacidad de introducir aspectos de interacción y comportamientos sociales en los modelos urbanos interactivos; se convierte entonces en una herramienta de simulación urbana incorporando al planificador, docente o estudiante en un nuevo mundo virtual que, paradójicamente ayudará a mejorar de manera significativa la realidad de su entorno.

\section{Conclusiones}

La metodología utilizada constituye una herramienta de apoyo en la planificación de espacios urbanos, en la que podrán elaborarse propuestas mediante simulaciones reales, destinada a mejorar el trabajo multidisciplinar entre el planificador y los usuarios.

Los entornos virtuales de las áreas objeto de estudio, serán de gran utilidad en todas las etapas de planificación y diseño urbano, incluyendo los procesos de toma de decisiones. Éstos permitirán describir y simular estados actuales y propuestos, con el objetivo de descubrir, pronosticar y prever comportamientos o estados futuros.

Finalmente, la metodología propuesta y los entornos virtuales de la Plaza Baralt generados, podrán ser utilizados en forma interactiva en cualquiera de las etapas de planificación y diseño urbano en espacios de carácter patrimonial, sirviendo como ejemplo en otros espacios con características similares. Además, puede servir de soporte en los procesos de revitalización de este espacio a través de la Intranet o del sito web.

La planificación urbana tiene que ser participativa para que tenga significado, conocer una situación y proyectarla en el tiempo y en el espacio, para enfrentar esa realidad futura.

\section{Agradecimientos}

A la Facultad de Arquitectura y Diseño de la Universidad del Zulia; y al personal que trabaja en el proyecto de investigación. 